University of Wollongong

Research Online

Faculty of Informatics - Papers (Archive)

Faculty of Engineering and Information

Sciences

$1-5-2006$

\title{
Undular bore solution of the Camassa-Holm equation
}

Timothy R. Marchant

University of Wollongong, tim@uow.edu.au

N. F. Smyth

University of Edinburgh, UK

Follow this and additional works at: https://ro.uow.edu.au/infopapers

Part of the Physical Sciences and Mathematics Commons

\section{Recommended Citation}

Marchant, Timothy R. and Smyth, N. F.: Undular bore solution of the Camassa-Holm equation 2006.

https://ro.uow.edu.au/infopapers/366

Research Online is the open access institutional repository for the University of Wollongong. For further information contact the UOW Library: research-pubs@uow.edu.au 


\title{
Undular bore solution of the Camassa-Holm equation
}

\author{
Abstract \\ Modulation theory is developed for a periodic peakon solution of the Camassa-Holm equation. An explicit \\ simple wave solution of these modulation equations is then derived; this simple wave describing the \\ evolution into an undular bore of an initial step. The characteristic on which the expansion fan occurs \\ (propagating at a nonlinear group velocity) has a turning point, illustrating the fact that there is a \\ minimum nonlinear group velocity at which the waves can propagate. A linear analytical solution, based \\ on an integral of the Airy function, is then derived to describe the evanescent portion of the undular bore \\ behind the turning point. Good agreement is found between the modulation theory plus Airy integral \\ solution and numerical solutions. \\ Disciplines \\ Physical Sciences and Mathematics

\section{Publication Details} \\ This article was originally published as: Marchant, TR \& Smyth, NF, Undular bore solution of the Camassa- \\ Holm equation, Physical Review E, 2006, 73(5), 057602. Copyright 2006 American Physical Society. The \\ original journal can be found here.
}




\title{
Undular bore solution of the Camassa-Holm equation
}

\author{
T. R. Marchant* \\ School of Mathematics and Applied Statistics, University of Wollongong, Wollongong, New South Wales 2522, Australia \\ N. F. Smyth ${ }^{\dagger}$ \\ School of Mathematics, The King's Buildings, University of Edinburgh, Edinburgh, Scotland EH9 3JZ, United Kingdom \\ (Received 29 June 2005; revised manuscript received 13 January 2006; published 4 May 2006)
}

\begin{abstract}
Modulation theory is developed for a periodic peakon solution of the Camassa-Holm equation. An explicit simple wave solution of these modulation equations is then derived; this simple wave describing the evolution into an undular bore of an initial step. The characteristic on which the expansion fan occurs (propagating at a nonlinear group velocity) has a turning point, illustrating the fact that there is a minimum nonlinear group velocity at which the waves can propagate. A linear analytical solution, based on an integral of the Airy function, is then derived to describe the evanescent portion of the undular bore behind the turning point. Good agreement is found between the modulation theory plus Airy integral solution and numerical solutions.
\end{abstract}

DOI: 10.1103/PhysRevE.73.057602

The Camassa-Holm $(\mathrm{CH})$ equation

$$
u_{t}-u_{x x t}+3 u u_{x}=2 u_{x} u_{x x}+u u_{x x x},
$$

has been much studied in the last decade. This interest is due to both its integrable nature and its unusual traveling wave solutions, such as the peakon, which is a soliton solution with a cusp at its peak (see Camassa et al. [1] and Camassa and Holm [2]). The interest in the peakon lies in its analogy with the wave of greatest height for surface water waves, see Lamb [3]. Alber et al. [4] used the methods of complex and algebraic geometry to obtain solutions of the $\mathrm{CH}$ equation in terms of angle representations. Special cases were considered, including finding the phase shifts for a two peakon interaction and a periodic traveling wave, termed a billiard solution. The billiard solution has cusped peaks and is a generalization of the peakon. They also wrote down the billiard solution in terms of a peakon series. Boyd [5] further examined series solutions for $\mathrm{CH}$ periodic waves and solitons, terming the billiard solutions as coshoidal waves. As well as the peakon series of Alber et al. [4] he obtained a Fourier series representation for the coshoidal wave. Johnson [6] found a parametric form for the general, implicit $\mathrm{CH}$ soliton solution and used computer algebra to derive the two and three soliton solutions.

Whitham [7] developed a modulation theory for the Korteweg-de Vries (KdV) equation based on the periodic cnoidal wave solution of the KdV equation with slowly varying parameters. These modulation equations were found to form a hyperbolic system of three first-order pde's for the (slowly varying) mean height, wave number, and amplitude of the modulated cnoidal wave. A useful, and easily derived, solution of the modulation equations for the $\mathrm{KdV}$ equation, derived by Gurevich and Pitaevskii [8] and Fornberg and Whitham [9], is a simple wave solution, which corresponds

\footnotetext{
*Electronic address: tim_marchant@uow.edu.au
}

${ }^{\dagger}$ Electronic address: N.Smyth@ed.ac.uk
PACS number(s): 05.45.Yv, 02.60.Lj, 47.35.-i

physically to an undular bore. This undular bore solution represents the evolution, and smoothing out, of an initial step in mean height.

In the present work the explicit coshoidal (or billiard) periodic wave solution is used as the basis for modulation theory and modulation equations describing slow variations in the mean height, wave number, and amplitude of the coshoidal wave are derived. The modulation equations are found to be hyperbolic and so an explicit undular bore solution of these modulation equations is found. The unusual feature of this undular bore solution is that the characteristics on which the simple wave solution occurs have a minimum velocity, so that finite amplitude waves occur at the trailing edge of the undular bore, rather than the usual case in which linear waves occur at the trailing edge.

Behind the trailing characteristic of the undular bore there is a region of exponentially decaying waves which is similar to the evanescent region behind the point at which the derivative of the group velocity vanishes in linear dispersive wave theory. A derivation similar, to that in Whitham [7], is used to obtain an Airy integral solution to describe the evanescent undular bore behind the turning point of the modulation theory. The undular bore solution and the Airy integral solution are then discussed and compared with numerical solutions of the $\mathrm{CH}$ equation.

The $\mathrm{CH}$ equation (1) has a special, peaked periodic wave solution, called a billiard solution or a coshoidal wave (see Alber et al. [4] or Boyd [5]). This coshoidal wave solution is

$$
u=[c / \cosh (\pi / k)] \cosh (\theta-\pi) / k,
$$

for $0 \leqslant \theta \leqslant 2 \pi$, where the phase speed $c=\omega / k$ is arbitrary and the phase $\theta=k x-\omega t$. This solution is extended periodically outside of the interval $0 \leqslant \theta \leqslant 2 \pi$. The cusps of the coshoidal wave occur at $\theta=0,2 \pi$ and their periodic extensions. The peak height of (2) is $c$ and the trough height is $c \operatorname{sech}(\pi / k)$. Hence the amplitude of the coshoidal wave is

$$
a=c[1-\operatorname{sech}(\pi / k)] .
$$

In the limit $k \rightarrow 0$, the coshoidal wave becomes the peakon $u=c e^{-|x-c t|}$. 
Modulation theory, as originally developed by Whitham [7], was based on averaging a Lagrangian for the governing equation. However, even though the $\mathrm{CH}$ equation (1) possesses a Lagrangian, it is easier to use the method of multiple scales and averaged conservation equations to obtain the relevant modulation equations. In terms of deriving modulation equations, the two techniques have been shown to be equivalent. For example, KdV modulation theory has been derived using a Lagrangian, see Whitham [7], and directly using multiple scales, see Myint and Grimshaw [10]. Also, Whitham [7] discusses the equivalence of the two techniques for various examples.

It should be noted that while the $\mathrm{CH}$ equation (1) is third order in $x$, the coshoidal wave solution (2) has only two free parameters, which means that (2) is a special case of the general periodic wave solution of the $\mathrm{CH}$ equation. The solution for this special case (2) is explicit, while the general traveling wave solution of (1) is implicit; see Johnson [6] for a derivation of the general, implicit, $\mathrm{CH}$ traveling wave solution. Hence we shall use the coshoidal wave (2) as the basis of our modulation theory as its derivation is then simple and straightforward. Moreover, it will be found that this restricted set of modulation equations gives a good description of a modulated peaked wavetrain. Details of the derivation of the full $\mathrm{CH}$ modulation equations can be found in Abenda and Grava [11].

The mass conservation equation for the $\mathrm{CH}$ equation is

$$
(\partial / \partial t)\left(u-u_{x x}\right)+(\partial / \partial x)\left(\frac{3}{2} u^{2}-\frac{1}{2} u_{x}^{2}-u u_{x x}\right)=0 .
$$

We now consider a slowly varying coshoidal wavetrain and wish to find equations for its slowly-varying parameters, the wave number $k$, frequency $\omega$, mean level $\beta$, and amplitude $a$. These parameters are functions of the slow space coordinate $X=\epsilon x$ and the slow time $T=\epsilon t$, where $0<\epsilon \ll 1$ is a measure of the slow variation. For a slowly varying wavetrain, the phase function is given by

$$
\theta=\epsilon^{-1} \Theta(X, T), \quad \omega=-\Theta_{T}, \quad k=\Theta_{X},
$$

where $\omega$ is the local frequency and $k$ is the local wave number, Whitham [7]. We now substitute the coshoidal wave solution (2) into (4), noting that $u=u(\theta, X, T)$, and average over a wave period $0 \leqslant \theta \leqslant 2 \pi$. This gives

$\partial \beta / \partial T+(\partial / \partial X)\left[\left(\omega^{2} / \pi k\right) \tanh \pi / k+\left(\omega^{2} / 2 k^{2}\right) \operatorname{sech}^{2} \pi / k\right]=0$,

where the mean wave height $\beta$ is

$$
\beta=\frac{1}{2 \pi} \int_{0}^{2 \pi} u d \theta=\frac{\omega}{\pi} \tanh \frac{\pi}{k} .
$$

The modulation equations for the modulated coshoidal wave are then completed by the wave conservation equation

$$
\partial k / \partial T+\partial \omega / \partial X=0 .
$$

At this point, the variables $x$ and $t$ rather than the slow variables $X$ and $T$ will be used for simplicity, as in Whitham [7].

The modulation equations (6) and (8) can be put in Riemann invariant form as

$$
\begin{gathered}
\omega=A_{+}[k \tanh (\pi / 2 k) / \tanh (\pi / k)] \text { on } C^{+}: \\
d x / d t=\omega / k+\pi \omega / k^{2} \sinh (\pi / k)+2 \pi \omega / k^{2} \sinh (2 \pi / k), \\
\omega=A_{-}[k / \tanh (\pi / 2 k) \tanh (\pi / k)] \text { on } C^{-}: \\
d x / d t=\omega / k-\pi \omega / k^{2} \sinh (\pi / k)+2 \pi \omega / k^{2} \sinh (2 \pi / k),
\end{gathered}
$$

where $A_{+}$and $A_{-}$are constants along the characteristics $C_{+}$ and $C_{-}$, respectively.

The centered simple wave solution of this system of hyperbolic modulation equations which corresponds to a physical initial condition is that along the characteristic (10) and corresponds to the dispersive resolution of an initial step down from $u=A$ to $u=0$. This centered simple wave solution is

$$
\begin{gathered}
\beta=(2 A / \pi) k \tanh (\pi / 2 k), \quad a=(\omega / k)(1-\operatorname{sech}(\pi / k)), \\
\omega / k=2 A[\tanh (\pi / 2 k) / \tanh (\pi / k)],
\end{gathered}
$$

where

$$
\frac{x}{t}=2 A \frac{\tanh (\pi / 2 k)}{\tanh (\pi / k)}\left[1-\frac{\pi}{k \sinh (\pi / k)}+\frac{2 \pi}{k \sinh (2 \pi / k)}\right] .
$$

The simple wave solution (11) can be explicitly evaluated by calculating $x / t, \omega, a$, and $\beta$ for specific values of $k$. Alternatively, if $x / t$ is chosen, then a transcendental equation must be solved for $k$.

At the leading edge of the expansion fan, the wave number $k \rightarrow 0$, which implies that peakons of amplitude $a$ $\rightarrow \omega / k=c=2 A$ propagate at the front of the bore, on a mean level of $\beta \rightarrow 0$. At the trailing edge of the expansion fan, the wave number $k \rightarrow \infty$, the mean level $\beta \rightarrow A$, the wave amplitude $a \rightarrow 0$, and the phase speed $c=\omega / k \rightarrow A$. Hence, at the trailing edge, the modulated wavetrain approaches a linear wavetrain, if the undular bore solution is extended back to zero amplitude (i.e., linear waves). The details of what happens at the trailing edge of the bore will be discussed and analyzed further below as the behavior there is more involved than for the $\mathrm{KdV}$ and other previously studied equations.

In this section the undular bore solution (11) will be compared with numerical solutions of the $\mathrm{CH}$ equation (1). The scheme used to find the numerical solutions is an implicit, three-level finite difference scheme with second-order accuracy. It is similar to the scheme developed for the BenjaminBona-Mahony (BBM) equation by Eilbeck and McGuire [12]. The parameters used for the numerical solutions were $\Delta x=2.5 \times 10^{-2}$ and $\Delta t=2.5 \times 10^{-5}$.

Figure 1 shows $u$ versus $x$ at $t=50$ for the step initial condition $u=A=1$ for $x<0$ and $u=0$ for $x>0$. Shown are the numerical solution and the wave envelope given by the simple wave solution of the modulation equations. The upper envelope is given by $\omega / k$, while the lower envelope is given by $(\omega / k) \cosh (\pi / k)$. An excellent comparison is obtained between the wave envelope of modulation theory and the numerical solution, throughout the bore. 


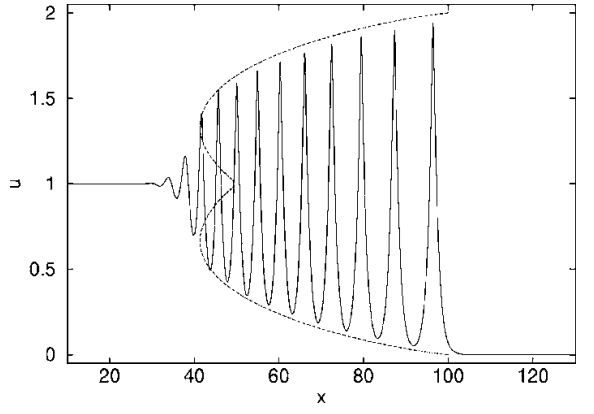

FIG. 1. $u$ versus $x$ at $t=50$. Shown are the modulation (dashed) and numerical (solid) solutions of (1). The modulation solution is the upper and lower envelopes of the simple wave solution (11).

An unusual property of the undular bore solution (11) is that the characteristics $C^{-}$(propagating with the nonlinear group velocity) have a turning point. The characteristics $C^{-}$ of the undular bore solution (11) then have a minimum slope (velocity) as a function of $k$ and so the characteristics of the simple wave cannot extend back beyond a fixed value of $x / t$. For $A=1$ this turning point occurs at $x / t=0.826$ for $k=2.39$ and is located at $x=41.3$ in Fig. 1. The simple wave solution is then only valid in front of the turning point. For the KdV equation and other nonlinear wave equations for which modulation theory has so far been developed, no turning point of the characteristics in the undular bore solution occurs. Figures 3 and 4 in Marchant and Smyth [13] show the development of undular bores which give the solution of initial-boundary value problems for the KdV equation. These $\mathrm{KdV}$ bores are typical; the amplitude of the waves in the bore decrease in a linear manner from the leading edge, where solitons occur, to the trailing edge, where linear waves of large wave number occur.

The $\mathrm{CH}$ modulation theory, however, describes a bore that varies from peakons at its leading edge to periodic waves with finite wave number of $k_{c}=2.39$ at its trailing edge, located at $x=41.3$ in Fig. 1. Hence, modulation theory predicts that no waves can propagate in the region $x<41.3$. It can be seen from Fig. 1 that the amplitude of the waves varies in an approximately linear manner near the front of the bore, but that the wave amplitude decreases rapidly to zero behind the turning point. Hence $\mathrm{CH}$ modulation theory accurately predicts that waves will only propagate in front of the turning point.

The group velocity $c_{g}=\omega^{\prime}$ for the linearized $\mathrm{CH}$ equation (12) has a turning point at $k_{m} \approx 1.73$, for which the corresponding minimum group velocity is $c_{g}=0.750$. Hence the linear dispersion relation predicts that no waves can propagate in $x<37.5$ in Fig. 1, which is fairly close to the turning point prediction from modulation theory. The reasons for the slight discrepancies between the modulation theory and numerical solutions at the trailing edge of the bore are related to the form of the coshoidal wave solution (2). This coshoidal wave reaches a mean level of $\beta=A$ in the long wave limit as $k \rightarrow \infty$. In this limit the wave amplitude also approaches zero. It might be thought that because the characteristics have a turning point at $k=k_{c}$, the simple wave solution should end with this wave number, rather than ex- tending to $k \rightarrow \infty$ as for the simple wave solution (11). Apart from the fact that if this were done, the resulting simple wave solution would not agree with numerical solutions; this is the wrong interpretation to put on what is happening at the turning point (see below for the derivation of the decaying solution behind the turning point).

Also, it should be noted that the occurrence of a minimum group velocity in (12) is due to the presence of the $u_{x x t}$ dispersive term. Hence, characteristics with a turning point should also occur for the BBM equation and other related equations which contain this type of dispersive term.

The simple wave (11) of modulation theory predicts peakons of amplitude two, at the leading edge. The height of the lead peakon in the numerical solution of Fig. 1 is 1.96. The modulation theory prediction is therefore extremely accurate. Interestingly, the amplitude of the lead soliton in a KdV bore (see Fornberg and Whitham [9]) and a magma bore (see Marchant and Smyth [14]) is also twice the height of the initial step.

In this section an analytical solution in the linear limit, which describes the evanescent region behind the modulation theory turning point, is derived, matched to the modulation theory solution and the resulting composite solution is then compared with full numerical solutions.

As the amplitude of the waves in the undular bore approach zero at the trailing edge of the bore, the waves in the evanescent region behind the turning point of the characteristics can be taken to have small amplitude. We hence set

$$
u=u_{0}+a e^{i(k x-\omega t)}, \quad \omega=u_{0} k\left[\left(3+k^{2}\right) /\left(1+k^{2}\right)\right],
$$

where $u_{0}$ is the mean level of the waves in the evanescent region, $a \ll 1$ and $\omega(k)$ is the linear dispersion relation for the $\mathrm{CH}$ equation. The linear dispersion relation (12) has a turning point with a minimum group velocity of $k_{m}=\sqrt{3}=1.73$. Expanding the linear dispersion relation in a Taylor series about $k=k_{m}$ gives

$\omega(k)=(3 \sqrt{3} / 2) u_{0}-\frac{3}{4} u_{0}\left(k-k_{m}\right)+\frac{3}{16} u_{0}\left(k-k_{m}\right)^{3}+\cdots$.

The general solution of the linearized $\mathrm{CH}$ equation can be written as a Fourier integral

$$
u(x, t)=\int_{-\infty}^{\infty} F(k) e^{i(k x-w(k) t)} d k,
$$

where $F(k)$ is related to the Fourier transform of the initial condition, see Whitham [7]. Substituting the dispersion relation expansion (13) into the Fourier solution (14) and rescaling $k$ gives

$$
u(x, t)=e^{i \theta_{1}} \int_{-\infty}^{\infty} F\left(k+k_{m}\right) e^{i\left(k x-\omega_{1}(k) t\right)} d k,
$$

where $\omega_{1}(k)=-\frac{3}{4} u_{0} k+\frac{3}{16} u_{0} k^{3}, \theta_{1}=\sqrt{3}\left(x-\frac{3}{2} u_{0} t\right)$. The phase of the wave at the turning point is $\theta_{1}=k_{m} x-\omega\left(k_{m}\right) t$ while the dispersion relation $\omega_{1}(k)$ is that for the linear $\mathrm{KdV}$ equation

$$
u_{t}-\frac{3}{4} u_{0} u_{x}+\frac{3}{16} u_{0} u_{x x x}=0,
$$

which has the similarity solution $u(x, t)=a_{1}+a_{2} \int_{-\infty}^{z} \mathrm{Ai}(s) d s$, where 


$$
z=\left(\frac{3}{4} u_{0} t-x\right) /\left(\frac{3}{16} u_{0}\right)^{1 / 3},
$$

and Ai is the Airy function (see Whitham [7]). Hence the full solution can be written in the form

$$
u=u_{0}+e^{i \theta_{1}}\left(a_{1}+a_{2}\right) \int_{-\infty}^{z} \operatorname{Ai}(s) d s .
$$

Taking the limit $x \rightarrow-\infty$ of the similarity solution (18) gives

$$
u=u_{0}+\left(a_{1}+a_{2}\right) e^{i \theta_{1}}, \quad x \rightarrow-\infty .
$$

Hence $u_{0}=1$ and $a_{2}=-a_{1}$ to satisfy the boundary condition of the initial step far behind the undular bore. The Airy integral solution needs to be matched to the periodic wave solution of modulation theory; this matching procedure should determine the unknown parameters $a_{1}$ in (18) and $A$ from the modulation theory solution. However, the linear solution (18) has a constant mean level $u_{0}=1$, which does not allow an accurate matching of the two mean levels as the modulation theory solution has a varying mean level. We then take $A$ $=1$ as this is the mean level of the modulation theory solution as $k \rightarrow \infty$ and we presume that this is the appropriate value which would be obtained by matching the modulation theory solution to a fully nonlinear evanescent solution, valid behind the turning point. It is noted that to allow the mean level to vary, the full nonlinear $\mathrm{CH}$ equation must be used behind the turning point.

The limit of (18) as $x \rightarrow \infty$ is

$$
u=u_{0}+a_{1} e^{i \theta_{1}}, \quad x \rightarrow \infty .
$$

So $a_{1}$ is the amplitude of the linear wavetrain, which then needs to be matched to the modulation theory solution. The coshoidal wave (2) is a nonlinear wavetrain, which can be expanded in a Fourier series to obtain the linearized wavetrain

$$
u=\beta+\left[2 \beta /\left(1+k^{2}\right)\right] \cos \theta+\cdots .
$$

The turning point in the modulation theory solution occurs at $k_{c}=2.39$, which gives a mean height of $\beta=0.877$ and a wave

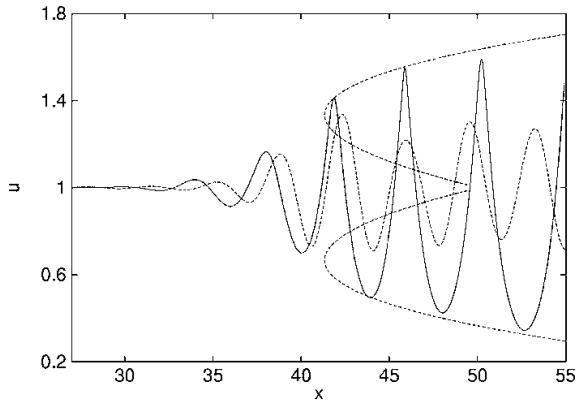

FIG. 2. $u$ versus $x$ at $t=50$. Shown are the modulation (dashed), Airy-integral (dashed), and numerical (solid) solutions of (1). The modulation solution is the upper and lower envelopes of the simple wave solution (11).

amplitude of 0.261. Comparing (20) and (21), matching then gives $a_{1}=0.261$. We note that the error between the mean levels of the two solutions, the modulation and linear evanescent solutions is quite small (cf. $\beta=0.877$ with $u_{0}=1$ ).

Figure 2 shows $u$ versus $x$ at $t=50$ for the step initial condition $u=A=1$ for $x<0$ and $u=0$ for $x>0$. Shown are the numerical solution and the upper and lower wave envelopes as given by the simple wave solution of the modulation equations. Also shown is the Airy integral solution (18). This figure shows the trailing edge of the undular bore featured in Fig. 1. The turning point of the modulation theory solution is at $x=41.3$, whilst the turning point of the linear $\mathrm{CH}$ dispersion relation is at $x=37.5$. For $x<37.5$ the surface of the bore is essentially flat, while for $x>41.3$ the solution is well described by modulation theory. The Airy integral solution (18) is seen to be an extremely accurate approximation to the numerical bore in the transition region $37.5<x<41.3$ between the two turning points. The wavelength and amplitude of the waves in this transition region are very accurately predicted by the Airy integral solution (18). There is a slight difference in the phase of the numerical and analytical waves, which is to be expected as modulation theory does not determine the phase of the wave.
[1] R. Camassa and D. D. Holm, Phys. Rev. Lett. 71, 1661 (1993).

[2] R. Camassa and D. Holm, Adv. Appl. Mech. 31, 1 (1994).

[3] H. Lamb, Hydrodynamics (Dover, New York, 1945).

[4] M. S. Alber et al., Lett. Math. Phys. 32, 137 (1994).

[5] J. P. Boyd, Appl. Math. Comput. 81, 173 (1997).

[6] R. S. Johnson, Proc. R. Soc. London, Ser. A 459, 1687 (2003).

[7] G. B. Whitham, Linear and Nonlinear Waves (Wiley, New York, 1974).

[8] A. V. Gurevich and L. P. Pitaevskii, Sov. Phys. JETP 33, 291 (1974).
[9] B. Fornberg and G. B. Whitham, Philos. Trans. R. Soc. London, Ser. A 289, 373 (1978).

[10] S. Myint and R. Grimshaw, Wave Motion 22, 215 (1995).

[11] S. Abenda and T. Grava, Ann. Inst. Fourier 55, 1001 (2005).

[12] J. C. Eilbeck and G. R. McGuire, J. Comput. Phys. 19, 43 (1975).

[13] T. R. Marchant and N. F. Smyth, Proc. R. Soc. London, Ser. A 458, 857 (2002).

[14] T. R. Marchant and N. F. Smyth, IMA J. Appl. Math. 70, 796 (2005). 